\title{
The Critically III Patients System Design Based on STM32
}

\author{
Jinjun Lu \\ School of Physics and Telecommunication Engineering, Shaanxi University of Technology, \\ Hanzhong, China \\ hsylu@163.com
}

Keywords: Embedded systems; STM32; Help patients; GSM; Wireless transmission

\begin{abstract}
This paper introduces the critically ill patients help system based on STM32F103RBT6 which can offer patients services in case of their immobilization during the illness. This kind of system is constituted by STM32, Wireless Unit, LCD monitor, GSM Unit and some peripheral units. Patients press the button when in need of help, then the corresponding service information through the wireless sending module is sent out and the receiver receives information and converts it into the alarm signals. Consequently LCD shows the demand of the patients, and it will be send to the nursing staff in the form of the messages by the module of GPRS. Thus, the patients will be given timely care and help. Experimental results show that the system has stable performance and high reliability which can meet the needs of patients. At the same time the system has characteristics of small volume, low cost and simple operation, suitable for families and small scaled hospitals. The application of the system can bring better economic benefits to the hospital, and it has a good value for popularization.
\end{abstract}

\section{Introduction}

With the continuous improvement of people's living standards and the rapid development of domestic and international service industries, people's demand for the service industry has becoming increasingly high. Meanwhile, the competition turns fairly fierce. At present, the majority of the domestic hospitals still use the wired call system, and in some small hospitals even the wired call system is unavailable which not only affects the hospital's reputation but also brings much inconvenience to the patients and their families [1]. Due to the disadvantages of the wired call system such as the complex wiring, expensive price, and immobility, the unattractive appearance and so on, the service quality has become unsatisfactory, and has been behind the time. Hospitals urgently need a convenient, high performance call system.

The critically ill patients help system based on STM32 are capable of sending alarm signals to the nursing staff in remote so as to improve the free activity and movement of the monitoring personnel and the convenience of the patients. Patients press the button when in need of help, then the corresponding service information through the wireless sending module is sent out and the receiver receives information and converts it into the alarm signals [2]. Consequently, LCD shows the demand of the patients, and it will be sent to the nursing staff in the form of the messages by the module of GPRS. Thus, the patients will be given timely care and help [3].

\section{Comparison and Selection of Design Schemes}

Host Selection. Scheme 1, using the STC89C52 chip as the main processor, which is an 8-bit microprocessor, has $256 \mathrm{~K}$ flash memory and $8 \mathrm{~K}$ programmable flash memory, but its high power consumption, limited resources I/O port, and running slow. Scheme 2, Main processor using STM32F103RBT6, it has an 32-bit microprocessor, with high performance, low power consumption, fast processing speed, I/O resource rich and so on.

Comprehensive comparison scheme 1 and 2, although the former is the main control system can be achieved, but taking into account the follow-up to communication and data transmission to the host computer, which needs high processing speed, so give up using STC89C52. The processor of the scheme 2 , is the use of $\mathrm{C}$ language programming, with the advantages of the programming 
modular design. Finally, the design of the system is realized by using the STM32F103RBT6 microprocessor which is used in the scheme 2.

Wireless Transceiver Module Selection. For the choice of wireless transceiver module, compared to the United States ACM's single emission chip ACMTX16, wireless amplitude modulation emission module YCF500-1 and China Taiwan PTC's PT2262 / PT2272, finally the design selected the PTC's PT2262/PT2272 chip to complete the wireless transceiver functions from the point of view of cost performance and ease of measurement.

\section{Hardware Design}

The system consists of wireless remote control handle transmitting module, wireless receiving circuit module, STM32 microprocessor module, LCD display module, sound and light alarm module and program download interface. According to the design of each unit circuit described above, considering the overall design of the system, the block diagram of the system is shown in Fig. 1.

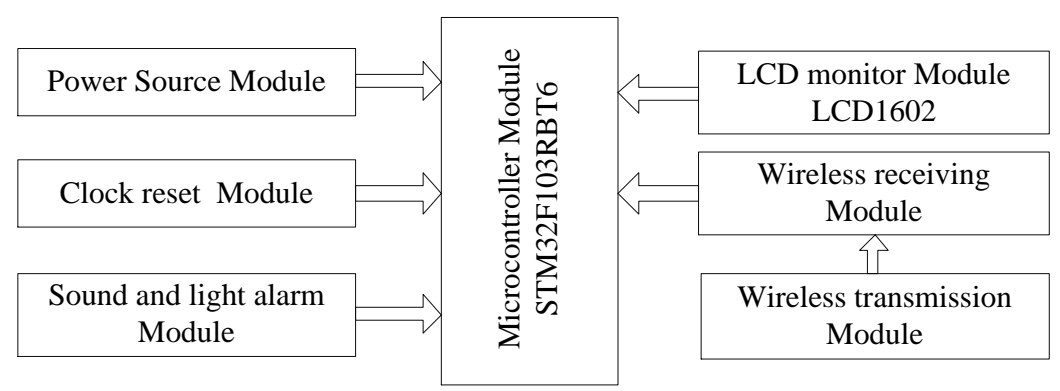

Figure 1. Block diagram of the system

Microcontroller Module. STM32F103RBT6, the STM32 family of processors based on ARM Cortex-M3 core, with low power, high integration degree, $72 \mathrm{MHz}$, 90DMIPS with 1.25 DMIPS/MHz, has a reset potential, low voltage detection, voltage regulator, dedicated $32 \mathrm{kHz}$ oscillator for RTC with calibration, and provide rich peripherals, 32-to-128Kbytes of Flash memory, serial wire debug (SWD) \& JTAG interfaces and USB interface, and also provides free easy to use development tools [4].

Wireless Transceiver Module. Wireless receiver, there are two, one is the super regenerative receiver, one is a super heterodyne receiver. The design uses the super heterodyne receiver. Transceiver system used by the transceiver module is 500 meters without shell $315 \mathrm{MHz}$ transmitter board two simplex $9805-2 \mathrm{~K}$ and $315 \mathrm{MHz}$ wireless super heterodyne type pin latch a wireless transceiver system is composed of receiver in 9903B transceiver system used in the transceiver module.

Encoding and Decoding Module. PT2262/2272 is a general low-power loss low-cost encoding and decoding circuit produced in Taiwan Princeton Technology Corp by CMOS manufacturing process. PT2262 may have 6-bit (D0-D5) data terminal pin, the set address and data code can send out in the serial way from the 17- pin it can be used for wireless remote transmitter circuit. The coding signals sent by the chip PT2262 are entire codon composed of address code, data code, and synchronization code. When the 17 pin is the low level, the $315 \mathrm{MHz}$ high- frequency transmission circuit will stop oscillating. Therefore, whether the high frequency transmission circuit will emit is completely controlled by 17-pin digital output of PT2262, completing amplitude shift keying (ASK modulation) is equivalent to $100 \%$ modulation of the amplitude [5].

Liquid Crystal Display Module. In the design, LCD1602 is used to show the serviced information of patients. This LCD has a display construction with 16 characters $\mathrm{x} 2$ lines which means it can display marks of two players at a same time [6].

Drip Feeding Call Module. The design uses photoelectric sensor to detect the liquid level signal of the liquid storage bottle. Parallel light beam emitted from the light emitting diode through the 
Murphy's dropper projected onto a photosensitive surface of the phototransistor, when there is no liquid phototransistor received the maximum illumination, photocurrent generated greatest, contrary phototransistor photocurrent generated reduced. In the case of low liquid level, the accumulation and movement of bubbles in the liquid surface are formed, and the divergence effect of parallel beam is obviously enhanced [7].

The alarm signal is sent out when the liquid level is too low and the bottle needs to be replaced or needs other assistance, this function is performed by the liquid storage bottle level detection circuit. Liquid Level detection means the infusion process has ended when the liquid level is below a predetermined position during the infusion. This time, the system sends an alarm signal and stops the infusion operations [8].

The schematic diagram of the liquid level detection circuit is shown in Fig. 2.

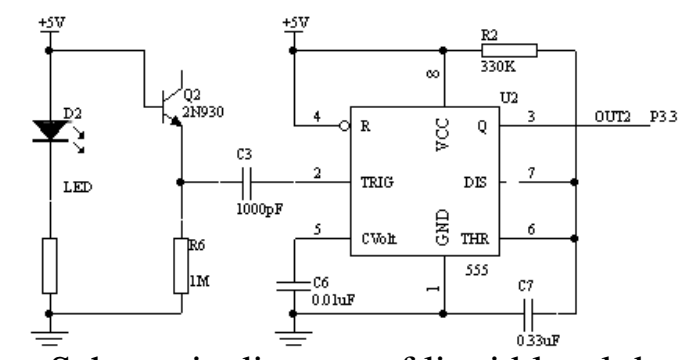

Figure 2. Schematic diagram of liquid level detection circuit

\section{Software Design}

Keil Introduction. This system software uses the $\mathrm{C}$ language, therefore this system uses Keil compilation of programming tools for application development and debugging. Keil company produced Keil is compatible with the microcontroller $\mathrm{C}$ language software development system, compared to the assembly, in the functional, structural, readability and maintainability, the $\mathrm{C}$ language has obvious advantages.

Keil advantages are: (1) Keil generated object code efficiency is very high, in which the assembly code generated is also very compact, and very easy to understand by the majority of the statement. (2) compared to the assembly, in functional, the structural, readability and maintainability, the $\mathrm{C}$ language has obvious advantages, so $\mathrm{C}$ language is not only easy to learn, but also very convenient to use. Experience in assembly language is only used in order to appreciate the advantages of the $\mathrm{C}$ language [9].

SMS Transceiver Control Software Design. This system is mainly to the GSM module to write different AT instructions through the STM32 microprocessor to complete the message sending. To send a message, first of all to understand the AT directive. The method of sending short message is also based on the AT command, through the control of several AT commands to complete the short message sending and receiving. The so-called "AT command" is the letters "AT" as a starting later joined a series of commands corresponding command character string, therefore, need to use the serial communication when the patient sends SMS messages.

Common AT Commands. The commonly used AT commands are as follows (at the end of each AT command by default with a newline, and the string "/r/n");

(1) AT

Handshake instruction, response to OK, showing the successful of the handshake.

(2) $\mathrm{AT}+\mathrm{CMGF}$

Select the message format. Use the AT $+\mathrm{CMGF}=[\langle$ mode $\rangle]$, indicating in PDU mode SMS and Unicode code form, supports Chinese when [<mode $\rangle]$ is 0 ; when $[\langle\operatorname{mode}\rangle]$ is 1 , it expressed in text mode SMS, ASCII code in plain English characters.

(3) AT+CMGS

Send a short message. AT + CMGS $=\mathrm{x}, \mathrm{x}$ is the length of the message to be sent [10].

System Ideas and Flowchart. Patients based on the STM32 help system is not to speak 
critically ill patients want to express their ideas through the button, and then sent to the phone to the patient's family by the GSM module. Family dependents of the patient through the short message can quickly help patients.

The design's purpose and requirements are:

(1) Software: The system have a beautiful interface, complete functions, On the basis of the optimal control algorithm, and can be made into software.

(2) Hardware: developed into a hardware control system with timely, accurate, reliable, simple and feasible, which will help to promote the advantages of the integrated circuit can be made to reduce the volume, easy storage and testing.

The design is controlled by buttons GSM module sends an SMS to the designated mobile phone, when the button is pressed, the serial port of the GSM module to send $\mathrm{AT}+\mathrm{CMGF}=1, \mathrm{AT}+\mathrm{CSCS}=$ "GSM", AT+CMGS=" +8613991614010", HELP, and 0X1A instruction. thereinto, AT+CMGF=1 is set for the properties to set the SMS text format, AT+CSCS= GSM " for the TE feature set, $\mathrm{AT}+\mathrm{CMGS}="+8613991614010$ "to send a set of specified phone number, HELP as the short message content; 0X1A the end flag, showing that the message content has been finished. The design flow chart is shown in Fig. 3.

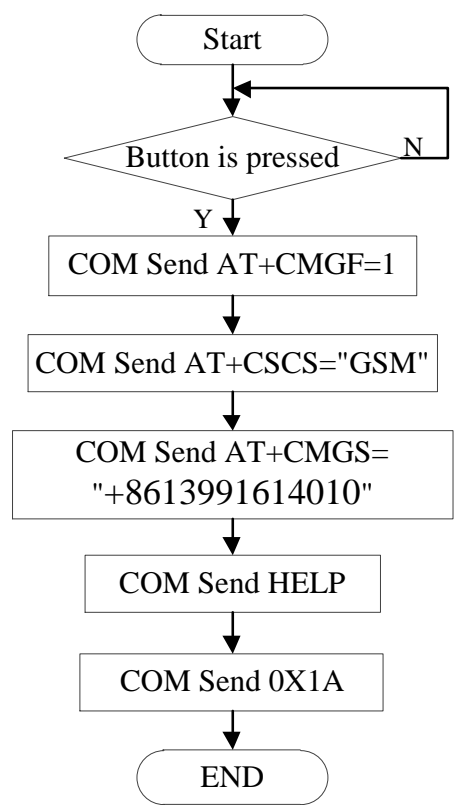

Figure 3. Design flow chart

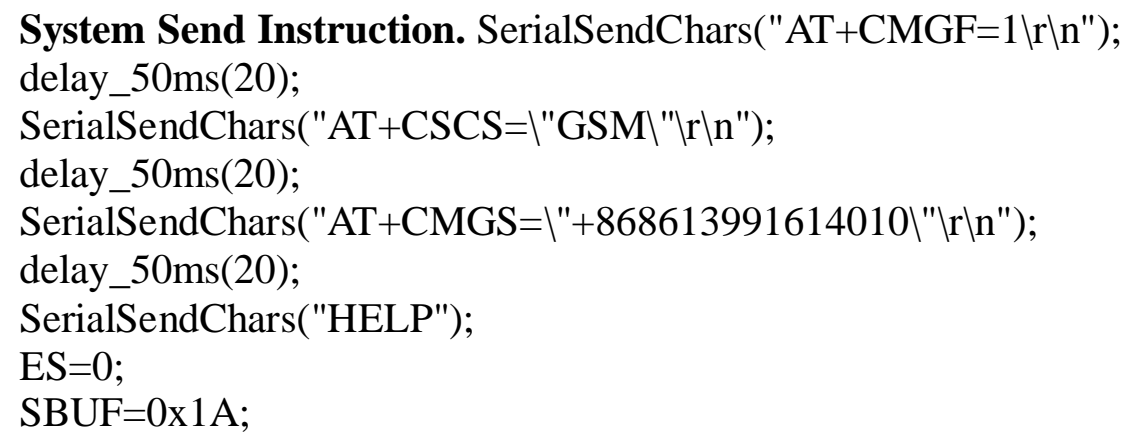

\section{Circuit Simulation and Physical Debugging}

Circuit Simulation. This circuit simulation uses Proteus to simulate. When pressing the P1.0 microprocessor STM32F103RBT6, sound and light alarm module's LED lights flashing, buzzer alarm music, the sound is loud and clear, while displaying "A Food" on the LCD display. The master controller is reset by pressing on the P1.1, the buzzer and the LED lights the same as the above case, the display "Bath B" on the LCD monitor. When Pressing the P1.2 or P1.3, the buzzer 
and the LED lights are normal, LCD displays are showing "C Drags" or "D Water". Therefore, the circuit simulation is quite successful.

Physical Debugging. After the production of hardware, software programming, the hardware and software to be combined on each module for debugging. The main three modules are LCD1602 module, sound and light alarm module, receiving and transmitting a wireless transceiver module.

Sound and light alarm circuit debugging: as long as the circuit of the buzzer is welded correctly, the wire is connected properly, then the alarm can be normal. Host power is turned on, the alarm circuit after writing the correct testing procedures and found that the circuit did not work properly. Disconnect the power supply, after careful examination, found that the 27, 28-pin of STM32 processor is connected upside down, test again after the changes, the buzzer alarm, while the lights flashing.

Debugging transceiver distance: The transceiver distance cannot meet the need, and module leaving only a position of antenna plus, the paint envelope to winding failed to solve the problem, because the STM32 family of processors work, will have a relatively strong electromagnetic radiation, the frequency is mainly in the $72 \mathrm{MHz} 900 \mathrm{MHz}$. It will affect the sensitivity of a wireless receiving module. The eventual adoption of a relatively thin cable to Pila straight welding obtain good results on the module.

\section{Summary}

Advantage of this design is that the portable device, low power, low cost, good stability, long distance wireless reception, alarm music is melodious. It brings a great activity space for patient monitoring, which is beneficial to the improvement of people's quality of life. It has a good application prospect, and has great practical value and popularization value.

\section{References}

[1] Arvind Bhimaraj. Remote Monitoring of Heart Failure Patients, Methodist DeBakey Cardiovascular Journal, Vol. 9 (2013) No.1, p.26-31.

[2] Rajan, Rajeev D. Wirelessly Enabled Remote Patient Monitoring Solutions, Medical Design Technology, Vol. 17 (2013) No.1, p.12-18.

[3] Stephen Agboola, Rob Havasy and Khinlei Myint-U. The Impact of Using Mobile-Enabled Devices on Patient Engagement in Remote Monitoring Programs, Journal of Diabetes Science and Technology, Vol. 7 (2013) No.3, p.623-629.

[4] Information on http://www.st.com/content/st_com/en.html

[5] Zhang P, Zhang K. A remote sleep-monitoring medical alarm system, Computer Communication Control and Automation (3CA), 2010 International Symposium on (IEEE, 2010). Vol. 1, p. 307-310. (In Chinese)

[6] Nurul Diyana Binti Johari. A Snooker Games Marking System (University Technologic Malaysia, Malaysia 2015), p.15.

[7] H.L. He, Y.X. Zhao and J. Qian. Realization of transfusion alarm system with pressure sensor, Chinese Medical Equipment Journal, Vol. 35 (2014) No.9, p.36-38.

[8] H.Q Yu, L.X Lu. Design and study of a common transfusion alarm and control system, Information of Medical Equipment, Vol. 19 (2011) No.9, p.10-12. (In Chinese)

[9] Information on http://www.keil.com/c51/

[10] S.R. Ptail, K.S. Warke. Communication of Different Mobile Phones Using Universal PC Suite, International Journal of Advanced Computational Engineering and Networking, Vol. 1 (2013) No.4, p.5-8. 